Acta, 6, 487, 1951; Bull. Soc. Chim. Biol,, 34, 1016, 1952; Experientia, 8, 317, 1952 .

[47] G. Biserte et L. Masse. C. R. Soc. Biol., 142, 664, 1948.

[48] R. Martin du $P_{A N}$, Les protéines du colostrum humain. Congrès International de Gynécologie et d'Obstétrique. Genève, 26 juillet 1954.

[49] P. Grabar. Bull. Soc. Chim. Biol., 36, 65, 1954.

[53] R. K. Morton. Biochem. Journal., 57, 231, 1954.

[54] P. Boulanger, G. Biserte et F. Courtot. Bull. Soc. Chim. Biol., 34, 366, 1952.

\title{
PROBLÈmES ACTUELS DE LA TECHNOLOGIE DES CONSERVES DE LAIT ( fin)
}

\author{
par \\ JEAN PIEN \\ Ingénieur Chimiste Docteur ès-Sciences \\ Directeur des Laboratoires de la Laiterie des Fermiers Réunis \\ Saint-Ouen (France)
}

\section{LES TECHNIQUES IDÉALES DE STERILISATION DU LAIT}

\section{$1^{\circ}$ La stérilisation en flux continu.}

Les données du problème portent, en elles-mêmes, la condamnation de principe de la stérilisation du lait dans les récipients d ə vente. La nécessité de stériliser le lait à haute température pendant un temps très court implique l'adoption de la stérilisation du lait liquide en flux continu.

Ce but peut être atteint de deux façons :

- Chauffer le lait "indirectement" en le faisant circuler, comme dans les pasteurisateurs classiques, entre des plaques ou entre deux tambours concentriques ou à l'intérieur de tubes (rectilignes ou en forme de serpentin) chauffés extérieurement par de la vapeur ou de l'eau chaude sous pression;

- Chauffer le lait "directement" par contact immédiat laitvapeur (soit par injection de vapeur dans le lait, soit par pulvérisation du lait dans une enceinte de vapeur).

Nous n'avons pas l'intention de décrire ici en détail les nombreux appareils, bien connus pour la plupart, qui permettent l'application de ces deux groupes de techniques. Nous nous contenterons d'énumérer les principaux d'entre eux et d'indiquer les avantages et les inconvénients des principes sur lesquels ils reposent. 
10 Chauffage indirect.

Les techniques de stérilisation continue par chauffage indirect sont, en réalité, très anciennes puisque les premières, à notre connaissance, remontent à 1912 .

Trois solutions sont à citer :

- Tambours chauffants concentriques (système Hecht-Tödt de Kiel, 1912, et système Beneke de la firme Rothenburg de Neumünster) ;

- Tubes chauffants en spirale (système Stork, Amsterdam et système Sturza de la firme Gerbig de Ludwigshafen) ou tubes rectilignes (système Silkeborg, Danemark et système Corblin, Paris);

- Plaques chauffantes conçues sur le même modèle que la plupart des pasteurisateurs classiques (système Bergedorf, AstraWerke de Hambourg, système Holstein et Kappert de Dortmund, système Alfa-Laval, etc...).

La plupart de ces appareils sont sommairement décrits dans l'ouvrage de ScHulz : Haltbare Milch [17].

Cette technique de chauffage indirect, pour la stérilisation continue, présente de sérieux inconvénients : la nécessité de porter le lait à $130-140^{\circ}$, et même davantage, implique la mise en curre de pressions élevées qui constituent une fatigue ou même un danger pour le matériel; la formation de dépôts sur ces surfaces portées à haute température est pratiquement inévitable et entraîne plusieurs conséquences : risques de caramélisation, surtout après un certain temps de fonctionnement, diminution de la qualité des échanges thermiques et baisse de température après 2 ou 3 heures de marche (obligeant à interrompre pour nettoyer), difficulté du nettoyage; enfin, danger d'obturation -.. (réellement constaté) surtout dans les systèmes tubulaires.

Ces critiques ne sont pas théoriques; nous les formulons à la lumière des enseignements de la pratique. Elles sont d'ailleurs confirmées par ScHulz [17]. Elles avaient déjà été formulées par LUECK et BrightoN en 1951 [18] qui s'expriment ainsi : "Les produits de faible acidité, comme le lait, doivent être portés rapidement à $130-150^{\circ}$ pour en effectuer la stérilisation en quelques secondes. Ces températures élevées excluent l'emploi des échangeurs à plaques des types existants. Les échangeurs tubulaires auxquels on a recours imposent des pressions de pompage élevées afin d'obtenir, dans le produit en circulation, la turbulance indispensable, d'une part à l'obtention d'un bon échange thermique, d'autre part à éviter autant que possible la formation de dépôts 
sur les surfaces portées à une température élevée. " (Notons que c'est généralement l’homogénéisateur qui, travaillant avant le stérilisateur continu, communique au lait la pression nécessaire, grâce à ses trois pompes à piston, décalées de $120^{\circ}$ d'arc entre les pistons pour réduire les pulsations. Néanmoins, ces hautes pressions de pompage n'évitent pas toujours la formation de dépôts, même dans les circuits tubulaires, avec tous les inconvénients qui en résultent.)

N'hésitons pas à dire que cette technique de chauffage indirect pour la stérilisation continue est loin de donner satisfaction : à notre avis, elle devra disparaître rapidement, pour céder la place à la technique de chauffage direct.

\section{$2^{\circ}$ Chauffage direct.}

Cette technique est récente, bien que le principe en soit connu depuis longtemps (1929) et encore très peu répandue.

Elle consiste à injecter de la vapeur à $160^{\circ}$ dans le lait lui-même pendant un temps extrêmement court et à refroidir immédiatement par expansion le lait ainsi stérilisé.

La réalisation industrielle la plus importante de ce principe est connue sous le nom d'upérisation et est due à l'Alpura de Berne (1952) [19] [20] [21] [22] [23].

Une autre solution industrielle du problème du chauffage direct est due à l'ingénieur français LAGUILHARRE. Le principe en est voisin de celui de l'upérisation : le lait est pulvérisé sous pression dans une chambre de vapeur maintenue à la température et à la pression voulues $\left(140-150^{\circ}\right)$ et immédiatement refroidi, comme dans l'upérisation, par détente sous vide [24] [25] [26].

Ces procédés présentent deux difficultés communes : le contact direct du lait avec la vapeur pose le problème de la propreté de la vapeur, et la légère condensation de vapeur dans le lait, suivie d'une concentration dans le stade de détente, pose le problème de la constance de l'extrait sec du lait. Ces deux difficultés sont résolues: la première par l'emploi d'un " transformateur de vapeur » permettant la production d'une vapeur "secondaire» irréprochable, la seconde par la mise en œuvre de dispositifs automatiques qui font que le léger mouillage qui se produit au cours de la stérilisation est très exactement compensé par la légère concentration qui intervient au moment du refroidissement instantané.

En revanche, cette technique présente des avantages nombreux et très importants :

- La surchauffe et la caramélisation sur une paroi sont impossibles;

- Il ne se forme pas de dépôts ; 
- Il est inutile d'interrompre la stérilisation pour procéder à des nettoyages;

- Le nettoyage est très facile ;

- Enfin, avantage majeur, le lait (refroidi brutalement dans le vide) est désaéré et désodorisé.

En fait, l'expérience montre que les laits stérilisés par "chauffage direct" sont absolument blancs, exempts de toute odeur et de toute saveur anormales. La supériorité de ces techniques sur les précédentes, en ce qui concerne le lait, est manifeste.

\section{$2^{\circ}$ Le remplissage aseptique.}

Les procédés de stérilisation que nous venons d'étudier sont done capables de conduire à du lait parfaitement stérile et exempt des inconvénients dont l'élimination constituait la donnée fondamentale du problème de la stérilisation du lait. Théoriquement, done, le problème est résolu.

Pratiquement, il reste encore un grand pas à franchir : le lait stérile qui sort en flux continu des appareils doit être conditionné dans les récipients de vente.

La solution idéale de ce problème nouveau consiste évidemment à remplir aseptiquement des récipients stériles.

Les techniques correspondantes sont assez nombreuses et bien connues, D'excellentes revues en ont été faites au cours du IIe Congrès International de la Conserve (Paris, 1951), notamment par Durocher et Roskis [27] et par Ball [28]. Il n'est donc pas nécessaire d'y revenir ici en détail. Rappelons seulement le nom des plus importantes d'entre elles : procédé H.C.F. (Heat-CoolFill) [29], [30], procédé Avoset, procédé Martin [31], [32], procédé Smith-Ball.

Les caractéristiques dominantes de ces techniques sont les suivantes :

- Elles conduisent à un remplissage qui peut être rigoureusement aseptique, ce qui revient à dire qu'elles apportent une solution techniquement parfaite du problème posé ;

- Elles comportent un équipement de remplissage et de fermeture généralement très compliqué et toujours fort coûteux ;

- Elles ne s'appliquent qu'au remplissage en récipients métalliques ou, exceptionnellement en bocaux de verre, mais ne semblent pas encore capables de permettre le conditionnement du lait stérile en bouteilles du type courant.

Sans vouloir entrer dans une étude plus détaillée de ces méthodes qui constituent, à coup sûr, avec la stérilisation continue, la véritable solution d'avenir du lait stérilisé, il faut loyalement recon- 
naître qu'elles ne sont pas encore applicables, encore moins généralisables dans le cas qui nous occupe ici — surtout si l'on songe que la majorité du lait stérilisé est actuellement conditionné en récipients de verre.

Pour que ces techniques puissent entrer dans la grande pratique industrielle du lait de conserve, il faudrait qu'un certain nombre de conditions nouvelles fussent remplies :

- Simplification de l'appareillage et de la technique ;

- Diminution sensible du prix de revient;

- Abandon de la bouteille de verre pour le lait stérilisé en faveur du conditionnement en récipient perdu (métallique ou autre) ;

- Adaptation des techniques de remplissage aseptique à l'utilisation de récipients non métalliques.

Nous savons que de sérieux efforts sont tentés en divers endroits en vue d'essayer de satisfaire à ces conditions. Mais les solutions définitives, simples, peu onéreuses, généralisables ne sont pas encore trouvées et ne semblent pas près de l'être.

Ainsi la solution idéale du problème du lait stérilisé liquide doit comporter deux étapes dont l'une est franchie (la stérilisation en flux continu) et dont l'autre (le remplissage aseptique) ne l'est pas encore au sens où nous l'entendons. Il en résulte que cette solution idéale appartient au domaine de l'avenir.

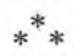

\section{LES TECHNIQUES INDUSTRIELLES ACTUELLES DE STÉRILISATION DU LAIT}

Devant cette situation, il est bien évident que la stérilisation du lait a dû, et doit, pour un certain temps encore, s'établir sur d'autres bases.

Etant, donné que la meilleure technique de stérilisation du lait est la stérilisation en flux continu, il convient d'essayer de la conserver. Mais, attendu que le conditionnement aseptique n'est pas encore applicable au cas des bouteilles de verre et probablement pas encore généralisable, quoique résolu, au cas des boîtes métalliques, il faut bien admettre l'hypothèse du remplissage non aseptique du lait stérilisé. Il en résulte immédiatement que le lait ainsi conditionné n'est pas stérile, même au sens industriel et pratique du mot. Une nouvelle stérilisation s'avère done nécessaire dans le récipient de vente... et l'on aboutit ainsi aux techniques généralement adoptées actuellement. Ayons le courage də dire que cette manière de faire est un non-sens théorique.

Mieux vaudrait, semble-t-il, abandonner la première stérilisation continue puisqu'elle est suivie d'une contamination, laquelle 
rend nécessaire une nouvelle stérilisation qui, cette fois, ne se produit pas "en flux continu " mais dans les récipients de vente, avec tous les inconvénients que cela entraîne pour le lait. On en reviendrait ainsi à la stérilisation unique - comme dans presque toute l'industrie des conserves.

En fait, le choix de la meilleure position à adopter mérite une discussion attentive que nous allons tenter de mener brièvement.

Nous avons rappelé, au début de cette étude, les inconvénients de la stérilisation classique du lait (altération des qualités organoleptiques et de la valeur alimentaire) résultant d'un chauffage trop prolongé, causé par l'inertie thermique des récipients. La stérilisation unique dans les récipients de vente - avec la sévérité du chauffage qu'elle doit comporter (notamment en ce qui concerne la durée) - conduit à ces altérations et à ces inconvénients.

Lorsque le lait a subi une première stérilisation effective en flux continu, tous les germes qu'il renfermait sont détruits. Mais la deuxième stérilisation qu'il doit subir en récipients de vente, lorsque le remplissage n'est pas aseptique, ne peut pas être comparée à l'unique stérilisation d'un lait brut. Il est évident que le nombre de germes à détruire dans une seconde stérilisation est infiniment moindre que celui du lait cru. Or il est bien démontré que la stérilisation peut être d'autant plus brève que la flore microbienne à détruire est moins abondante. Les résultats de VAN DER STEUR, par exemple, ont montré que si le nombre de germes vivants est mille fois plus faible, la durée de chauffage à $115^{\circ}$ peut être 3 à 4 fois plus courte, toutes choses égales d'ailleurs [33]. Il n'est pas douteux que, dans un matériel propre et bien désinfecté, les contaminations que peut subir le lait entre les deux stérilisations sont très faibles et n'ont absolument rien de commun avec la flore microbienne initiale du lait cru.

Dans les techniques généralement appliquées à l'heure actuelle, la seconde stérilisation peut donc être beaucoup moins sévère que la stérilisation unique du lait brut et ne présente pas, par conséquent, les mêmes inconvénients. C'est la raison pour laquelle, à la question de savoir si la première stérilisation ne devrait pas être supprimée lorsque le conditionnement ne peut pas être aseptique, il convient de répondre de la manière suivante :

Dans la mesure où le lait n'est pas fortement recontaminé entre les deux stérilisations, la première doit être conservée pour permettre à la seconde d'être brève et de ne pas entrâ̂ner de graves inconvénients en ce qui concerne les caractères organoleptiques du lait final.

L'expérience a pleinement confirmé ce point de vue et c'est pourquoi les techniques actuelles - en dépit du fait qu'elles sont 
très loin d'être idéales et rationnelles - sont néanmoins valables, pratiquement, et sont largement répandues.

Nous n'entrerons pas ici dans le détail de ces techniques. Rappelons en seulement le principe commun qui découle clairement de ce qui précède :

1. Le lait cru est homogénéisé et subit une première stérilisation qui est effective (le lait est parfaitement stérile). Cette opération, appelée improprement " préstérilisation ", est réalisée en flux continu et la meilleure formule consiste en un chauffage direct, c'est-à-dire du type upérisation. (Malheureusement, e'est en général par chauffage indirect que l'on procède, et c'est là, avons-nous dit, une erreur.)

2. Le lait stérile ainsi obtenu est véhiculé dans des pompes, canalisations, cuves pour aboutir au remplissage à chaud en bouteilles ou en boîtes métalliques, propres et pratiquement stériles. Si le matériel est bien nettoyé et désinfecté - et si les opérations sont conduites avec une asepsie suffisante, le lait ainsi conditionné est très peu chargé en microorganismes.

3. Le lait conditionné, légèrement recontaminé, est soumis à une stérilisation finale, improprement appelée "post-stérilisation " qui doit et qui peut lui conférer la stérilité absolue ou du moins une stérilité pratique suffisante pour assurer une conservation très prolongée. Les appareils utilisés dans ce but devraient permettre un chauffage de courte durée et sont généralement constitués par des "stérilisateurs continus" de modèles divers, dont les plus répandus en Europe sont actuellement les stérilisateurs à colonne de pression d'eau, système français Pierre Carvallo, système anglais WEBSTER, système hollandais STORK, qui sont tous bien connus et ont été maintes fois décrits [17], [27], [34], [35], [36].

A quels résultats pratiques conduisent ces techniques?

Nous avons dit qu'elles n'étaient pas rationnelles, mais qu'elles étaient pratiquement valables. Cela signifie qu'elles permettent d'atteindre une conservation suffisante, mieux, qu'elles conduisent le plus souvent à la stérilité totale. Mais il faut bien reconnâ̂tre que les caractères organoleptiques du lait sont encore nettement altérés. La condition de rapidité et de brièveté du deuxième chauffage n'est jamais suffisamment remplie pour qu'il en soit autrement. Le lait obtenu est toujours légèrement teinté et présente toujours une odeur et une saveur plus ou moins prononcées de "cuit" de "caramel " ou même parfois de "brûlé ". Ces inconvénients, certes, sont nettement moins marqués que dans le cas d'une stérilisation unique (donc plus longue) du lait dans les récipients de vente qui sont presque toujours des flacons de verre - mais ils ne sont pas 
nuls. On aboutit, en somme, à une solution intermédiaire entre les solutions anciennes, qui faisaient du lait stérilisé un produit inconsommable, et les solutions idéales dont nous avons parlé plus haut.

C'est pourquoi nous n'hésitons pas à dire que les techniques. actuelles de stérilisation du lait ne résolvent pas parfaitement le problème et qu'elles devront disparaître tôt ou tard.

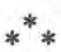

\section{LES SOLUTIONS D'AVENIR}

Nous avons déjà dit en quoi elles devront consister : stérilisation du lait en flux continu par chauffage direct (type upérisation) suivie du remplissage aseptique. Nous savons aussi pourquoi les difficultés et le prix de revient actuel du deuxième stade de cette solution en retarderont l'avènement.

Une question se pose donc : en l'absence des solutions idéales et devant l'imperfection des méthodes actuelles, que faut-il espérer pour l'avenir immédiat?

Avant de répondre à cette question, nous devons ouvrir une parenthèse.

L'histoire de la stérilisation du lait, au cours de ces vingt dernières années, a subi une évolution bien curieuse : les inconvénients de la stérilisation classique appliquée au lait (inconvénients que nous avons rappelés au début de cette étude) étaient bien connus et de nombreuses tentatives ont vu le jour dans le but d'améliorer les techniques classiques de la conserverie en vue de leur application au cas du lait. Cette amélioration, de toute évidence, devait consister en une réduction du temps de séjour des récipients dans les autoclaves. Depuis longtemps, des progrès avaient été réalisés par l'adoption de la rotation de l'autoclave ou des paniers à l'intérieur de l'autoclave. Il est inutile de rappeler ici les nombreuses solutions fondées sur ces principes.

Or, en 1936-1938, CaRvallo et nous-même, avons entrepris l'étude systématique dé la pénétration de la chaleur dans les boîtes métalliques contenant du lait, tournant individuellement autour de leur axe, et avons abouti à cette conclusion qu'il existait, pour chaque cas particulier, une vitesse de rotation optima qui permettait les échanges les plus rapides. Nous avons, par exemple, montré qu'une boîte $4 / 4$ remplie de lait à $80^{\circ}$ et, tournant sur son axe à 94 tours/minute dans une enceinte de vapeur à $126^{\circ}$, atteignait, dans toute sa masse, la température de $125^{\circ}$ en 3,5 minutes et qu'un tel lait, préalablement contaminé massivement de sporulés aérobies, était stérile dans ces conditions (1). Enfin, ce lait ne présentait

(1) Résultats non publiés. 
aucune coloration et aucune saveur de cuit ou de brûlé (le refroidissement, immédiatement réalisé sous pression dans l'autoclave, était extrêmement rapide.)

De ces études, qui ont comporté de nombreuses déterminations expérimentales, est sorti le premier autoclave continu CARVALLO [37] [38], dans lequel les boîtes entraient, par l'intermédiaire d'un sas rotatif, dans un grand autoclave où elles étaient soumises, du fait de leur avancement par roulement sur des plans inclinés, à un mouvement de rotation autour de leur axe. Un premier refroidissement sous pression avait lieu dans un compartiment clos de l'appareil. Le refroidissement final s'effectuait à la pression atmosphérique. Dans son principe, cet appareil est donc assez analogue au stérilisateur bien connu de la Food Machinery and Chemical Corporation (F.M.C. Stérilisator) à tambour rotatif et piste hélicoïdale montée sur la périphérie, et au stérilisateur anglais MATHER et Platt.

Un perfectionnement de l'appareil CARvallo (qui ne semble pas applicable aux deux autres) consisterait à dissocier les deux mouvements d'avancement et de rotation des boîtes, de manière à permettre de modifier le débit de l'appareil sans changer la vitesse de rotation (et inversement) et surtout de construire des appareils beaucoup moins encombrants puisque le temps de séjour ne serait plus lié au nombre de boîtes entrant dans l'autoclave à l'unité de temps. Cette modification, qui semblerait devoir apporter un gros progrès dans la réalisation des autoclaves continus a été brevetée mais n'a pas encore fait l'objet de réalisations industrielles [39].

Nous devons nous empresser d'ajouter que ces divers appareils ne permettent pas la stérilisation en bouteilles de verre puisque les récipients, dès qu'ils ont franchi le sas d'entrée, sont introduits brusquement dans une enceinte de vapeur à haute température. Même dans le cas des bouteilles remplies et fermées à $80^{\circ}$ (ce qui constitue déjà une difficulté) l'écart de température à subir brutalemeñt par ces récipients serait de l'ordre de 40 à $45^{\circ}$. Un tel écart serait trop grand pour les bouteilles de verre, sauf s'il s'agissait de verres spéciaux à faible coefficient de dilatation. Au reste, l'emploi de bouteilles de verre ne permettrait pas les échanges thermiques rapides dont nous avons parlé et le résultat obtenu, en ce qui concerne les caractères organoleptiques du lait, serait certainement beaucoup moins bon que dans le cas des boîtes métalliques.

Quoi qu'il en soit, ce stérilisateur CARvallo de 1936-1937, constituait, à coup sûr, un très grand progrès en matière de stérilisation du lait. La deuxième guerre mondiale est venue interrompre l'épanouissement de cette technique nouvelle et nous avons assisté, dans les années qui ont suivi la guerre, à une évolution toute dif- 
férente dans le sens des "techniques actuelles» dont nous avons parlé plus haut.

La possibilité de réaliser une stérilisation unique en récipients (de préférence métalliques en raison de la rapidité de la transmission de la chaleur) agités vigoureusement n'a pas été perdue de vue par les constructeurs.

A ce sujet, il convient de signaler que le stérilisateur-refroidisseur continu "Sterilmatic" de la Food Machinery and Chemical Corporation, déjà cité, permet, dans ses modèles actuels, d'imprimer aux boîtes des vitesses de rotation élevées, et par conséquent d'effectuer la stérilisation en des temps très courts. D'autre part, nous savons qu'un appareil de conception nouvelle est actuellement en construction en France ; dans ce stérilisateur, où les boîtes vont tourner à 120 tours/minute, elles ne séjourneront que 3,5 à 4 minutes dans une atmosphère à $250^{\circ}$. Les conditions que Carvallo et nous-même avions préconisées en 1938 sont ainsi réalisées et les résultats obtenus avec un appareil expérimental selon ce nouveau principe montrent que les qualités organoleptiques du lait sont intégralement respectées. Il est prévu que ce nouveau stérilisateur sera en fonctionnement en France à l'automne prochain.

Il nous semble maintenant possible de répondre à la question que nous posions au début de ce chapitre : que faut-il espérer pour l'avenir immédiat?

En attendant l'avènement des "techniques idéales" (stérilisation en flux continu et remplissage aseptique) nous pouvons songer à améliorer les " techniques actuelles» de la façon suivante : la deuxième stérilisation (en récipients de vente) gagnerait à être traitée par l'une de ces techniques que nous venons de décrire et qui consistent à assurer la rotation optimale des récipients dans l'autoclave. Or, les stérilisateurs continus à colonnes de pression d'eau ne se prêtent pas, sous leur forme actuelle, à cette rotation provoquée. C'est pourquoi nous pensons que cette "post-stérilisation" devrait être réalisée dans un appareil à rotation individuelle des récipients (à pistes rectilignes comme dans le Carvallo, à pistes hélicoïdales comme dans le F.M.C. ou le Mather et Platt, ou, mieux encore, à mouvements dissociés dans un appareil nouveau à créer). Le cas particulier des récipients de verre demanderait une solution spéciale : verre à faible coefficient de dilatation et utilisation d'étuis métalliques perforés pour permettre le guidage sur les rampes.

La seconde stérilisation, ainsi améliorée par l'adoption d'un temps de séjour dans l'autoclave ramené à quelques minutes, conduirait à du lait qui, en récipients métalliques, ne présenterait aucun défaut (l'expérience l'a prouvé) et, en récipients de verre, 
serait évidemment de qualité très supérieure à celui que l'on obtient actuellement.

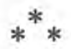

\section{RÉSUMÉ}

L'auteur consacre son exposé à une étude des problèmes du lait stérilisé non concentré, qui domine actuellement la question des conserves du lait.

Le lait étant un milieu de culture excellent pour de nombreux microbes, y compris les sporulés les plus résistants, et son $p$ H étant voisin de la neutralité, sa stérilisation exige un traitement thermique énergique; d'où le risque des modifications indésirables de la couleur, de la saveur et de l'odeur, et de la valeur alimentaire.

La connaissance du mécanisme de ces altérations est importante pour la recherche des meilleures solutions techniques.

Pour ce qui concerne le brunissement du lait sous l'effet du chauffage, les travaux de BuRTon ont montré qu'il est linéairement proportionnel à la durée du chauffage et que le coefficient de température de la réaction est égal à 2,44 (lait non concentré). Par ailleurs, le coefficient de température de la destruction des spores dans le lait est, d'après les travaux de $R_{A H N}$ et de Clegg, de l'ordre de 9 (8 à 10). La comparaison, par un raisonnement mathématique simple de ces deux réactions, permet de confirmer (ce que l'expérience a constaté depuis longtemps) qu'il y a intérêt, pour éviter le brunissement, à mettre en œuvre des températures élevées pendant un temps aussi bref que possible.

Les modifications de la saveur et de l'odeur surviennent en général avant le brunissement et paraissent provenir de deux réactions différentes : caramélisation et libération de $\mathrm{H}^{2} \mathrm{~S}$. Elles dépendent des conditions de chauffage de manière analogue au brunissement, et les mêmes conclusions leur sont applicables.

La valeur alimentaire est affectée par les mêmes réactions qui conduisent au brunissement : blocage de certains acides aminés indispensables (lysine, puis arginine et histidine), qui deviennent inaccessibles à la digestion.

A la suite de ces considérations, l'auteur fait une revue eritique des méthodes de stérilisation utilisées actuellement, pour se tourner vers l'avenir : à son avis, e'est au chauffage en flux continu par contact direct avec la vapeur, suivi du remplissage aseptique, qu'il faut donner la préférence - technique qui n'a toutefois pas encore trouvé une solution réellement satisfaisante.

A défaut, après avoir rappelé ses propres études et celles de Carvallo sur la stérilisation du lait en boîtes tournant rapidement sur leur axe, l'auteur estime que l'application de ces techniques, notamment si les mouvements de rotation et de translation des boîtes peuvent varier indépendamment l'un de l'autre, sont à même de conduire, surtout avec les récipients métalliques (avec ou sans stérilisation préalable, en flux continu) à un lait exempt de défauts. 


\section{BIBLIOGRAPHIE}

[1] R. J. Ramsay, P. H. Tracy et H. A. Ruehe. Journal Dairy Sci., 26, $17,1933$.

[2] K. M. Henry, S. K. Kon, C. H. LeA et J. C. D. White. Journal Dairy Res., 15, 292, 1948.

[3] C. H. LeA. Journal Dairy Res., 15, 369, 1948.

[4] C. H. Lea et R. S. Hannan. Biochim. Biophys. Acta, 3, 313, 1949 ; 4, 518,$1950 ; 5,433,1950$.

[5] C. H. LeA. Le brunissement non enzymatique. C. $R$. du II ${ }^{\mathrm{e}}$ Congrès Intern. de la Conserve. Paris, octobre 1951.

[6] F. H. Grimbleby. Journal Dairy Res., 21, 207-211, 1954.

[7] H. Burton, D. M. Akam, C. C. Thiel, E. Grinsted et L. F. L. Clegg. Journal Soc. Dairy Techn., 6, 98-113, 19:3.

[8] H. Burton. Journal Dairy Res., 21, 194-203, 1954.

[9] O. RaHN. Bact. Rev., 9, 1, 1, 1945.

[10] L. F. L. ClegG. Journal Soc. Dairy Tech., 3, 4, 238, 1950.

[11] N. P. TARAssuk et H. D. Simonsen. Food Techn., 4, 88, 1950.

[12] L. T. Winger. U.S. Patent, 2, 596-753. Voir D.S. A., 14, 756, 1952.

[13] A. R. Patton, E. G. Hill et E. M. Foreman. Science, 105, 481, 1947 ; 107, 623, $1948 ; 108,659,1948$.

[14] R. J. Evans et H. A. Butrs. Journal Biol. Chem., 178, 543, 1949.

[15] K. M. Henry et S. K. Kon. Biochim. Biophys. Acta, 5, 455, 1950.

[16] R. R. Baldwin, J. R. Lowry et R. Thiessen. Food Research, 16, $107,1951$.

[17] M. E. Schulz. Haltbare Milch. Edit. Hans Carl, Nüremberg, 1954.

[18] R. H. LueCK et K. W. BRIGHton. C. R. du II Congrès international de la Conserve. (Paris, octobre 1951).

[19] Société Alpura, Berne (Suisse). Brevet Suisse ne 284.061 du ler novembre 1952. Voir Milchwissenschaft, 8, 139, 1953.

[20] H. Mohler. Chimia, 6, 212, 1952.

[21] E. Zolitkofer. Neue Zürcher Zeitung, 29 octobre 1952.

[22] P. Hatrry. Mitt. a. d. Gebiete Lebensm. u. Hyg., 44, 159, 1953.

[23] A. Fuchs. Mitt. a. d. Gebiete Lebensm. u. Hyg., 44, 163, 1953.

[24] Anonyme. Le Lait. Mars 1956.

[25] Anonyme. Milchwissenschaft, 11-12, 1953.

[26] Anonyme. Dairyman, 71, 18, 1954.

[27] J. Durocher et G. Roskis. $C, R$. du IIe Congrès International de la Conserve. Paris, octobre 1951.

[28] C. O. BALL. C. R. du IIe Congrès International de la Conserve. Paris, octobre 1951.

[29] C. O. Ball, U.S. Patent, 2.029.303, 4 février 1936.

[30] C. O. BALL. Food Research, 3, 1938.

[31] W. M. Martin. Food Industries, 20, 6, 71, 1948.

[32] W. M. MARTin. Canner, 111, 12, 22, 24, 1950.

[33] J.P. K. VAN DER Steur. C. R. du II e Congrès International de la Conserve. Paris, 1951.

[34] Anonyme. Officiel de la Conserve, 14, 32, 1949. 
[35] W. Stonk. Molk. u. Käserei-Ztg., 2, 1062, 1950.

[36] Journées Scientifiques du lait stérilisé. Ann. de la Nutrition et de l'Alimentation 9, 2, 1955.

[37] Brevet français $n^{\circ} 824.07 \mathrm{I}$ du 15 octobre 1936.

[38] Brevet français $n^{\circ} 829.138$ du 10 novembre 1936 .

[39] Brevet français $n^{\circ} 844.428$ du $1^{\text {er }}$ avril 1938.

\section{BIBLIOGRAPHIE ANALYTIQUE}

\section{LES LIVRES}

Agenjo Cecilia (C.). - Encyclopédie du lait (en espagnol). 1 vol. relié, 995 pages, 28x20, 418 figures. Editeur : Espasa-Calpe. Madrid, 1956.

La littérature consacrée au lait vient de s'enrichir d'un ouvrage imposant dont le titre est à lui seul tout un programme, et dont l'A. est bien connu des lecteurs de cette Revue, autant par ses divers articles que parl'analyse de ses livres précédents.

Il est évidemment très difficile de rendre compte en quelques mots de cette nouvelle présentation, en raison de la documentation considérable qu'elle contient.

La première partie de l'ouvrage est consacrée à la production du lait. Il est ainsi d'un grand intérêt d'y trouver d'abord un chapitre consacré aux pâturages (composition, valeur...), puis un second relatif à l'alimentation des vaches laitières, pour laquelle l'A. exprime en un tableau les besoins des animaux selon la quantité et la qualité du lait produit, puis les suivants ayant trait au rendement laitier, à la sélection, aux croisements, et enfin des directives qui doivent présider à la gestion de l'étable, tout cela faisant l'objet d'importants développements que l'on suit aisément sur le plan pratique. Le chapitre 6 est consacré, comme il se devait, aux maladies animales, et en particulier à la mammite.

Cette dernière partio se termine par un chapitre d'une valeur particulière, consacrée à la production du lait de chèvre, et portant la signature du Professeur Rafael Saraza Ortiz, qui traite le problème dans son ensemble avec la maîtrise et la compétence qui le caractérisent : considérations zootechniques, physiologiques, technologiques, donnent au sujet exposé toute sa valeur.

La deuxième partie est réservée à la sécrétion lactée, à la traite, au ramassage et au transport du lait, puis aux maladies transmissibles à l'homme par le lait et ses dérivés, et enfin, à l'utilisation du lait tant en Espagne qu'en le reste du monde.

La chimie et la bactériologie des produits laitiers, la composition du lait et ses caractéristiques biochimiques et physicochimiques sont ensuite développées, en visant le lait des différentes femelles laitières utilisées.

Nous voudrions pouvoir parler longuement du chapitre consacré à la science des industries laitières (coagulation, fabrications consécutives, 